\title{
La investigación médica en el Instituto Mexicano del Seguro Social: siete décadas de trabajo, logros y retos
}

Moisés Mercado, ${ }^{1}$ Javier Torres, ${ }^{2}$ Fernando Guerrero-Romero, ${ }^{3}$ Mardia López-Alarcón ${ }^{4}$ y Héctor Mayani ${ }^{5 *}$ ${ }^{1}$ Unidad de Investigación Médica en Enfermedades Endocrinas, Ciudad de México; ${ }^{2}$ Unidad de Investigación Médica en Enfermedades Infecciosas y Parasitarias, Ciudad de México; ${ }^{3}$ Unidad de Investigación Biomédica de Durango, Durango; ${ }^{4}$ Instituto Mexicano del Seguro Social, Coordinación de Investigación en Salud, Unidad de Investigación Médica en Nutrición, Ciudad de México; ${ }^{5}$ Unidad de Investigación Médica en Enfermedades Oncológicas, Ciudad de México. Instituto Mexicano del Seguro Social, Coordinación de Investigación en Salud, México

\section{Resumen}

Desde su fundación, hace más de 75 años, el Instituto Mexicano del Seguro Social (IMSS) realiza investigación multidisciplinaria -biomédica, clínica y epidemiológica- enfocada a entender y resolver los problemas médicos que aquejan a sus derechohabientes (más de 50 \% de la población mexicana). En un inicio, la investigación fue resultado de esfuerzos individuales y aislados. En la década de 1960, un número reducido de investigadores conformó los primeros grupos de investigación. Actualmente, 240 científicos de tiempo completo trabajan en cinco centros y 40 unidades de investigación ubicados en distintos estados de México. Además, aproximadamente 270 médicos efectúan investigación clínica en las distintas unidades de primer, segundo y tercer nivel de atención. Durante estas siete décadas, los científicos del IMSS han realizado aportaciones relevante para la medicina, las cuales no solo han ayudado a incrementar el conocimiento acerca de la etiopatogenia de numerosas enfermedades, sino también al diagnóstico, pronóstico y tratamiento de ellas. En este artículo se presenta un panorama general sobre la investigación médica que se desarrolla en el IMSS, a partir de un enfoque histórico y de la revisión de algunas de las contribuciones más relevantes en los distintos campos de la investigación.

PALABRAS CLAVE: IMSS. Investigación biomédica. Investigación clínica. Investigación epidemiológica.

\section{Medical research at the Mexican Institute of Social Security: Seven decades of work, achievements, and challenges}

\section{Abstract}

Since its foundation, more than 75 years ago, the Mexican Institute of Social Security (IMSS) has carried out multidisciplinary research -biomedical, clinical and epidemiological-focused on understanding and solving the medical problems that afflict its beneficiaries (more than $50 \%$ of the Mexican population). Initially, research was the result of individual and isolated efforts. In the 1960s, a small number of researchers formed the first research groups. Currently, 240 full-time scientists work at five centers and 40 research units located in different states of Mexico. In addition, approximately 270 doctors carry out clinical research at different primary, secondary and tertiary care units. During these seven decades, IMSS scientists have made relevant contributions to medicine, which have not only helped increase knowledge on the etiopathogenesis of numerous diseases, but also their diagnosis, prognosis and treatment. This article presents an overview of medical research carried out at IMSS, based on a historical approach and a review of some of the most relevant contributions in different fields of research.

KEY WORDS: IMSS. Biomedical research. Clinical research. Epidemiological research.

Correspondencia:

*Héctor Mayani

E-mail: hectormayaniv@gmail.com

DOI: 10.24875/GMM.21000389
Gac Med Mex. 2021;157:470-478

Disponible en PubMed

www.gacetamedicademexico.com CC BY-NC-ND (http://creativecommons.org/licenses/by-nc-nd/4.0/). 


\section{Introducción}

A principios del siglo XX, la esperanza de vida en el mundo era de 31 años; actualmente es de 72 años.' Dicho incremento es, en gran medida, resultado de los avances en la medicina, que, a su vez, son producto de la investigación. La investigación médica comprende, al menos, tres tipos de investigación: biomédica, clínica y epidemiológica. La investigación biomédica está enfocada a entender y caracterizar mecanismos y procesos a nivel genómico, genético, molecular, celular y fisiológico. El sujeto de estudio puede ser un gen, una proteína, una célula o un proceso. En la investigación clínica, el sujeto de estudio es el individuo. Este tipo de investigación incluye estudios enfocados a la prevención, diagnóstico o tratamiento de una enfermedad. Finalmente, en la investigación epidemiológica, el sujeto de estudio puede ser desde un grupo pequeño de personas hasta poblaciones enteras. A través de ella se busca entender patrones, causas, factores de riesgo y formas de controlar las enfermedades.

Desde su fundación, hace más de 75 años, el Instituto Mexicano del Seguro Social (IMSS) ha llevado a cabo investigación multidisciplinaria - biomédica, clínica y epidemiológica- enfocada a entender y resolver los problemas médicos que aquejan a sus derechohabientes. En este artículo presentamos un panorama, desde nuestra perspectiva, sobre la investigación médica que se desarrolla en el IMSS, a partir de un enfoque histórico y de la revisión de algunas de las contribuciones más relevantes en los distintos campos de la medicina.

\section{El Instituto Mexicano del Seguro Social}

Fundado en 1943, el IMSS es la institución de salud más grande de México; ofrece atención a más de 69 millones de personas, lo que corresponde a $55 \%$ de la población. A lo largo del territorio nacional dispone de 1527 unidades de medicina familiar, 249 hospitales de segundo nivel de atención y 25 unidades médicas de alta especialidad. El número de personas que labora en el IMSS sobrepasa el medio millón, de los cuales, aproximadamente $50 \%$ corresponde a personal de atención a la salud.

\section{Perspectiva histórica de la investigación en el IMSS}

Incipientemente, la investigación en el IMSS inició a finales de la década de 1940. A partir de la iniciativa y esfuerzos de los doctores Ignacio Morones Prieto y Bernardo Sepúlveda, la investigación en el IMSS comenzó como una labor complementaria a las actividades asistenciales. Durante esos primeros años, la actividad científica consistió en esfuerzos aislados llevados a cabo en distintos hospitales, sin que hubiera articulación entre ellos. Fue con la creación del Centro Médico Nacional, en 1961, que se propició un ambiente de investigación que favoreció la interacción de diversos grupos. Así surgió la primera línea de investigación en biología de la reproducción. Uno de los productos más importantes de esa línea de trabajo fue el desarrollo de una "minipíldora" anticonceptiva, basada en una dosis reducida de progestágeno. ${ }^{2}$

Con la intención de integrar y fortalecer la investigación científica en el IMSS, en 1966 se creó el Departamento de Investigación Científica, por iniciativa del doctor Jorge Martínez Manatou, quien fue su primer director. Dicho Departamento se integró con la participación de ginecólogos, endocrinólogos, patólogos y bioquímicos. Posteriormente, se incorporaron especialistas en inmunología, farmacología y genética.

Los proyectos de investigación fueron financiados con fondos del propio IMSS y de la Fundación Ford. Comenzó, entonces, la compra de equipos y abastecimiento de materiales para integrar la infraestructura propicia que hiciera factible el desarrollo de los distintos proyectos de investigación. Una de las primeras adquisiciones fue un microscopio electrónico, equipo que simbolizó el inicio de la investigación como una actividad de importancia para la institución. Durante los siguientes años, el número de grupos de investigación en el IMSS fue aumentando a la par que la investigación se consolidó. El Departamento de Investigación Científica se convirtió en la Coordinación Nacional de Investigación Médica y, posteriormente, se transformó en la Coordinación de Investigación en Salud (CIS).

A lo largo de todo ese tiempo, médicos sobresalientes, comprometidos con el desarrollo de la investigación, dirigieron el Departamento de Investigación Científica, la CNIC y la CIS. Entre ellos se encuentran los doctores Carlos Beyer, Juan García, Fernando Flores, Arturo Zárate, Ignacio Madrazo, Onofre Muñoz, Homero Martínez, Dante Amato y Fabio Salamanca. Cabe mencionar que durante la gestión del doctor Onofre Muñoz, la presencia de centros y unidades de investigación en el IMSS se extendió a diferentes estados del país, lo que contribuyó a que hoy en día 
se tenga una amplia cobertura nacional de grupos de investigación. Por otra parte, es importante destacar la labor pionera que desempeñaron los doctores Roberto Kretschmer, Armando Isibasi, Luis Benítez, Salvador Armendares, Fabio Salamanca, Salvador Villalpando, Marcos Velasco, Francisco Velasco, Jesús Kumate, Arturo Zárate, Xavier Lozoya, José Sánchez Corona, Salvador Saíd, Alfredo Feria y Pablo Hernández, entre otros.

\section{Estado actual}

Actualmente, la investigación en el IMSS se desarrolla en 40 unidades y cinco centros de investigación, distribuidos a lo largo del país. La institución cuenta con 240 investigadores que se dedican de tiempo completo a la actividad científica y aproximadamente 270 médicos que dedican tiempo parcial a realizar investigación clínica o epidemiológica. De los poco más de 500 investigadores con los que cuenta el IMSS, aproximadamente $70 \%$ pertenece al Sistema Nacional de Investigadores.

Las actividades de investigación en salud son dirigidas, fomentadas y normadas por la CIS, encabezada actualmente por el doctor César Raúl González Bonilla. Anualmente, esta comunidad científica publica más de 800 artículos científicos en revistas especializadas y gradúa alrededor de 100 alumnos de maestría y doctorado en diversas áreas de la salud. Problemas prioritarios como la obesidad, la diabetes, el cáncer y las enfermedades infecciosas emergentes, entre otros, se abordan de manera multidisciplinaria y multicéntrica, mediante colaboraciones intra y extrainstitucionales, en las que participan instituciones nacionales y extranjeras. La infraestructura y el conocimiento en tecnologías de punta, como la genómica, la biología celular, la microscopia electrónica y la citometría han permitido que algunas de las unidades y centros de investigación ofrezcan pruebas diagnósticas y de pronóstico que los laboratorios clínicos no pueden realizar, lo que repercute en beneficio de miles de pacientes.

\section{Contribuciones al conocimiento médico.}

Durante los últimos 70 años, en el IMSS se ha realizado investigación de calidad e impacto en diversas áreas del conocimiento médico. Para los fines de este trabajo destacamos, como ejemplo, algunas de esas contribuciones.

\section{Genética}

La genética, en particular la citogenética, es una de las áreas que se empezó a desarrollar importantemente en el IMSS desde 1960. En 1971, Armendares, Salamanca y Frenk, investigadores del Hospital de Pediatría del Centro Médico Nacional, observaron la presencia de alteraciones cromosómicas en niños (uno a 60 meses de edad) que presentaban desnutrición severa. ${ }^{3}$ Este trabajo, publicado en la revista Nature, fue uno de los primeros a nivel mundial en demostrar alteraciones cromosómicas estructurales como consecuencia de la nutrición deficiente y estableció las bases para el desarrollo de un programa sobre estudios genéticos en la población infantil mexicana. Este mismo grupo de investigación identificó diversas alteraciones en el número y estructura cromosómica en pacientes pediátricos. .,5 $^{4}$ Paralelamente, el grupo encabezado por José María Cantú y José Sánchez Corona, del Centro Médico de Occidente, en Guadalajara, describió los hallazgos citogenéticos de la entidad conocida como síndrome de Cantú (MIM No. 239850), caracterizado por hipertricosis, macrosomía al nacimiento y diversas alteraciones faciales y anatómicas. ${ }^{6} \mathrm{Al}$ día de hoy, se han reportado más de 30 casos en la literatura médica internacional.

\section{Oncología}

Por su alta incidencia y mortalidad, el cáncer constituye una de las áreas prioritarias de investigación en el IMSS. Debido a su elevada frecuencia, en México y en el mundo, el cáncer cervicouterino ha sido el foco de atención prioritaria de diversos grupos científicos del IMSS y se han realizado numerosos estudios multicéntricos a nivel nacional. A través de dichos estudios, se ha determinado la frecuencia de los subtipos del virus del papiloma humano (VPH), agente causal de dicha neoplasia, y se ha demostrado que sus patrones de frecuencia y distribución son distintos a los reportados en otras regiones del mundo. ${ }^{7}$ Considerando que se cuenta con vacunas comerciales que se aplican ampliamente en la población general, este hallazgo tiene implicaciones importantes. En otro estudio multicéntrico, se observó que las coinfecciones con distintos genotipos de VPH parecen desempeñar un papel "protector" en mujeres infectadas. ${ }^{8}$

Durante los últimos años, el cáncer gástrico se ha convertido en un problema oncológico en el mundo. Las tasas más altas de mortalidad por este tipo de 
neoplasia ocurren en dos regiones del mundo: Asia y Latinoamérica, siendo la infección por Helicobacter pylori el principal factor de riesgo. La investigación realizada en la Unidad de Investigación Médica (UIM) en Enfermedades Infecciosas y Parasitarias ha contribuido al entendimiento de la fisiopatología de esta enfermedad, al identificar mutaciones o alteraciones en la expresión genética en los pacientes que padecen este tipo de cáncer.9,10 Lo anterior ha contribuido a la identificación de marcadores útiles para la prevención o para diagnóstico temprano del cáncer gástrico.

En cuanto a neoplasias hematológicas, trabajos realizados en la UIM en Enfermedades Oncológicas han contribuido al entendimiento de la biología de diferentes tipos de leucemias. Este grupo reportó que, en la leucemia mieloide crónica, el tratamiento con IMATINIB (fármaco inhibidor de la proteína BCR-ABL, característica de este tipo de leucemia) elimina a la gran mayoría de las células leucémicas, pero no a las células iniciadoras de la neoplasia, las cuales no solo sobreviven, sino que pueden expandirse en cultivos in vitro; ${ }^{11}$ hallazgo con implicaciones en el tratamiento de este tipo de leucemia. Este mismo grupo demostró que las células estromales mesenquimales de la médula ósea de pacientes con síndrome mielodisplásico, considerado como un estadio preneoplásico, presentan alteraciones genéticas y funcionales, lo cual puede tener implicaciones en la respuesta al tratamiento. ${ }^{12}$ Este estudio fue uno de los primeros en demostrar que no solo las células hematopoyéticas son anormales en algunas neoplasias hematológicas, sino que el microambiente de la médula ósea también se encuentra alterado. Recientemente, el grupo de oncoinmunología del Centro de Investigación Biomédica de Oriente (CIBIOR), en Puebla, reportó que la cortactina (una proteína que se une a la actina y participa en procesos de adhesión y migración celular) desempeña un papel relevante en la fisiopatología de la leucemia linfoblástica aguda infantil. Esta proteína participa en la migración tisular y en la recaída de la enfermedad, por lo que es un marcador diagnóstico y pronóstico para esta enfermedad..$^{13}$

Los adenomas pituitarios constituyen uno de los tipos más comunes de tumores intracraneales. En la mayoría de los casos se presentan de forma esporádica y su etiopatogenia es poco conocida. El grupo de investigación de la UIM en Enfermedades Endocrinas recientemente demostró, a través de un estudio transcriptómico y epigenético, que este tipo de tumores puede tener tres orígenes celulares distintos, de acuerdo con el factor de transcripción que determina su diferenciación. ${ }^{14}$ Este hallazgo puede contribuir al desarrollo de terapias más eficaces para este tipo de neoplasias.

\section{Microbiología e inmunología}

Debido a la importancia de Helicobacter pylori como el principal factor de riesgo para cáncer gástrico, en la UIM en Enfermedades Infecciosas y Parasitarias se han realizado estudios multicéntricos y multinacionales para entender la adaptación evolutiva de la bacteria a las diferentes poblaciones humanas en América ${ }^{15}$ El enfoque genómico de estos estudios aporta información de gran relevancia que ayudará a entender la fisiología de esta bacteria, su interacción con el ser humano y para explicar las diferencias en el riesgo de cáncer gástrico existentes entre los distintos países americanos. ${ }^{16}$

La tuberculosis representa una de las enfermedades infecciosas más frecuentes en el mundo. La susceptibilidad a esta enfermedad depende, en gran medida, de la respuesta del sistema inmune innato ante la infección por micobacterias. La Unidad de Investigación Biomédica de Zacatecas ha demostrado que LL-37, un péptido antimicrobiano, desempeña un papel muy importante en este proceso, ya que actúa como inmunomodulador de macrófagos durante la infección por Mycobacterium tuberculosis, controlando la producción de citocinas proinflamatorias y antiinflamatorias..$^{17}$ La caracterización de la fisiología de Mycobacterium tuberculosis y de la respuesta inmune ante esta infección contribuirá al desarrollo de esquemas terapéuticos más eficaces.

Uno de los grupos líderes en el área de la inmunología ha sido, sin duda, el de la UIM en Inmunoquímica. Dicho grupo ha trabajado durante más de 30 años en el estudio de la respuesta inmune ante infecciones bacterianas y virales, con miras al desarrollo de vacunas. Encabezado por el doctor Armando Isibasi, este grupo encontró que las porinas, proteínas localizadas en la membrana externa de Salmonella typhi, bacteria responsable de la fiebre tifoidea, pueden ser el blanco para generar protección inmune contra ese patógeno. ${ }^{18}$ Las porinas también han mostrado actividad protectora en humanos..$^{19} \mathrm{~A}$ partir de estas observaciones, este grupo de investigación ha trabajado en el desarrollo de una vacuna contra la fiebre tifoidea y otros tipos de salmonelosis.

Por otra parte, el grupo de la UIM en Inmunología estudia el papel de la hormona prolactina como 
agente inmunomodulador. Los trabajos de este grupo han demostrado que la prolactina participa en la activación de linfocitos T CD4 y linfocitos B, promoviendo la producción y secreción de interleucina-2 e interferón-gamma, ${ }^{20}$ así como las implicaciones fisiológicas de la prolactina en distintas condiciones nosológicas.

\section{Nutrición, síndrome metabólico, obesidad y diabetes}

La lactancia materna tiene efectos importantes sobre el desarrollo y crecimiento de los niños. Estudios en la UIM en Nutrición demostraron que la lactancia materna reduce la frecuencia y severidad de infecciones respiratorias y gastrointestinales, y protege el crecimiento de los lactantes, permitiéndoles el crecimiento en percentiles semejantes al de lactantes de países desarrollados. ${ }^{21,22}$ También han demostrado que intervenciones en la etapa neonatal basadas en la suplementación con ácido docosahexaenoico (DHA) atenúan la respuesta inflamatoria mediada por interleucina-1 $\beta$, la evolución clínica y protege el estado nutricio de neonatos con sepsis. ${ }^{23}$

La obesidad y el síndrome metabólico en las etapas pre y puberal son cada vez más prevalentes y su detección y tratamiento oportunos son cruciales para prevenir el desarrollo de comorbilidades cardiovasculares y cerebrovasculares en etapas posteriores de la vida de estos pacientes. Estudios realizados en la UIM en Nutrición acerca de la prevalencia de trastornos como dislipidemias, resistencia a la insulina y síndrome metabólico, han demostrado que la composición de ácidos grasos poliinsaturados de la dieta y de las membranas celulares, así como la suplementación con DHA, pueden tener impacto en la disminución de la resistencia a la insulina, la dislipidemia y la hiperandrogenemia en las niñas. ${ }^{24-27}$

En la UIM en Epidemiología Clínica del Hospital de Especialidades y en la UIM en Bioquímica del Centro Médico Nacional Siglo XXI se llevan a cabo estudios de colaboración para definir la epidemiología molecular de la obesidad y de la diabetes en México. Entre estos, destacan estudios de colaboración con instituciones nacionales y extranjeras en los que se ha identificado la asociación entre polimorfismos de un solo nucleótido de ciertos genes, como $A B C A 1$ y MGEAS-14, y la susceptibilidad para desarrollar diabetes tipo $2,,^{28,29}$ así como la asociación entre el gen del receptor de melanocortina ${ }^{30,31}$ y el desarrollo de obesidad en niños y adultos mexicanos. En la actualidad, se considera que la obesidad y la diabetes tipo 2 son condiciones proinflamatorias. En esta área del conocimiento, investigadores de las unidades de investigación en Enfermedades Endocrinas, en Epidemiología Clínica y en Bioquímica, han realizado estudios experimentales y clínicos sobre el perfil inflamatorio en la obesidad y la diabetes. ${ }^{32-35}$

La obesidad mórbida constituye un problema de salud creciente, cuyo manejo requiere un manejo multidisciplinario. En 2012, se creó la Clínica de Obesidad Mórbida del Hospital de Especialidades del Centro Médico Nacional Siglo XXI, en la que convergen el esfuerzo conjunto de los servicios de endocrinología, medicina interna y cirugía y de la UIM en Enfermedades Endocrinas. Esta clínica multidisciplinaria, sin duda la más grande de México, constituye una fuente importante de información clínica y de material biológico que ha permitido la realización de varios estudios, tanto clínicos como biomédicos. Entre estos destacan el seguimiento a largo plazo de pacientes sometidos a cirugía bariátrica, ${ }^{36}$ la definición del perfil proinflamatorio de pacientes con obesidad mórbida, ${ }^{37}$ la determinación del papel del receptor de la hormona de crecimiento en la distribución corporal del tejido adiposo ${ }^{38}$ y un estudio sobre la prevalencia de apnea obstructiva del sueño en personas con obesidad mórbida. ${ }^{39}$

Investigadores de la Unidad de Investigación Biomédica de Durango han estudiado, durante casi 20 años, el papel del magnesio en la obesidad, la hipertensión, la diabetes y el síndrome metabólico. A través de estudios clínicos controlados, demostraron que el uso de suplementos de este oligoelemento mejora prácticamente todos los componentes del síndrome metabólico, tanto en niños como adultos. ${ }^{40-43}$ Otra contribución de este grupo de investigadores es el desarrollo del índice triglicérido/glucosa como un biomarcador confiable y de bajo costo para el escrutinio de resistencia a la insulina. ${ }^{44}$

Finalmente, es importante mencionar que si bien la diabetes tipo 1 es considerablemente menos frecuente que la diabetes tipo 2, investigadores clínicos y biomédicos del IMSS han hecho importantes contribuciones que abarcan la inmunopatogénesis de esta enferme$\operatorname{dad}^{45}$ y la demostración de que quienes la padecen son portadores también de síndrome metabólico. ${ }^{46}$

\section{Enfermedad renal crónica}

Desde hace más de 20 años, la UIM en Enfermedades Nefrológicas ha sido reconocida como 
uno de los grupos líderes de México en el estudio de la enfermedad renal crónica. Una de las aportaciones más importantes de este grupo es la demostración, mediante un estudio clínico que incluyó a cerca de 1000 pacientes, de que la depuración de creatinina no es un predictor apropiado de mortalidad en personas con insuficiencia renal terminal bajo tratamiento sustitutivo. Este hallazgo propició un cambio drástico en la prescripción de diálisis, orientándola hacia una valoración clínica integral y no a un valor numérico, como la depuración de urea. En un estudio paralelo en pacientes en hemodiálisis se obtuvieron resultados similares. ${ }^{47,48}$ Estudios posteriores en la misma Unidad demostraron que la sobrecarga de líquidos se asocia a inflamación, ${ }^{49,50}$ desnutrición, disfunción sistólica y diastólica del miocardio ${ }^{51,52}$ y a mayores tasas de mortalidad, comorbilidades y costos, ${ }^{53}$ especialmente en pacientes con diabetes, en quienes el peritoneo no permite una ultrafiltración adecuada.

\section{Aterogénesis, enfermedad cardio y cerebrovascular}

Durante los últimos 10 años, investigadores de la UIM en Trombosis, Hemostasia y Aterogénesis, en colaboración con especialistas clínicos, tanto del Centro Médico Nacional Siglo XXI como del Centro Médico Nacional La Raza, han realizado importantes investigaciones traslacionales sobre la fisiopatología de la disfunción endotelial y la susceptibilidad a desarrollar enfermedad cardio y cerebrovascular. ${ }^{54-56}$ Dentro de estos, destacan dos estudios publicados recientemente. El primero demuestra la existencia de polimorfismos de un solo nucleótido de los genes que codifican la enzima convertidora de angiotensina y el angiotensinógeno, que específicamente se asocian a riesgo de eventos cerebrovasculares isquémicos en pacientes mexicanos. ${ }^{57}$ El segundo explora polimorfismos de un solo nucleótido de los genes AOX, LPA, MMP9 y TPO y su relación con el aumento en la susceptibilidad de aterotrombosis en adultos mexicanos. ${ }^{58}$

\section{Enfermedades neurológicas}

Una de las aportaciones más sólidas de la UIM en Enfermedades Neurológicas es el tratamiento farmacológico de la neurocisticercosis subaracnoidea con praziquantel. ${ }^{59}$ Por otro lado, quizá la contribución mejor conocida (y también la más polémica) de los neurocientíficos del IMSS es el tratamiento de la enfermedad de Parkinson avanzada con autotrasplantes de médula suprarrenal. ${ }^{60,61}$ En esta misma Unidad se llevan a cabo estudios, experimentales y clínicos, sobre la fisiopatología y el tratamiento quirúrgico y farmacológico de la lesión medular traumática. ${ }^{62-64}$

\section{Contribuciones a la atención del paciente}

Además de las numerosas contribuciones en la generación de conocimiento científico, que se refleja en la publicación de artículos trascendentes en revistas de alto factor de impacto y con múltiples citaciones, el trabajo que se realiza en los distintos Centros y Unidades de Investigación del IMSS ha contribuido importantemente en la atención al paciente. A continuación presentamos algunos ejemplos representativos de lo anterior.

La UIM en Nutrición ha trabajado en la descripción de la composición de la leche humana y el tiempo de adecuación de la lactancia exclusiva. Sus hallazgos forman parte de un documento guía publicado por la Organización Mundial de la Salud. Junto con el Hospital de Pediatría del Centro Médico Nacional Siglo XXI, esta Unidad ha colaborado en el establecimiento y manejo del lactario (banco de leche), que se utiliza principalmente en pacientes hospitalizados en la Unidad de Cuidados Intensivos Neonatales. Por otra parte, ha colaborado en la introducción de fórmulas lácteas enriquecidas al Cuadro Básico de Alimentos del IMSS.

La UIM en Enfermedades Nefrológicas ha trabajado en el diseño e implementación de programas de diálisis peritoneal en pacientes con insuficiencia renal crónica. También ha contribuido a las guías internacionales de diálisis peritoneal, las cuales fueron publicadas por la Organización Mundial de la Salud. Igualmente, su contribución incluye el diseño de estrategias tanto para la optimización de recursos en pacientes que reciben terapia sustitutiva renal (diálisis peritoneal y hemodiálisis), como para la reducción de la tasa de infección peritoneal en pacientes con insuficiencia renal crónica tratados con diálisis peritoneal. En la UIM en Epidemiología Clínica del Hospital de Especialidades del Centro Médico Nacional Siglo XXI, se han desarrollado programas y guías institucionales para la prevención, diagnóstico y tratamiento integral de la diabetes, obesidad y síndrome metabólico. Otra de sus contribuciones ha sido la optimización de recursos para la prevención, diagnóstico y tratamiento de los trastornos metabólicos. Esta Unidad tiene interacción y colaboración con unidades de medicina 
familiar en la prevención, diagnóstico y tratamiento de los trastornos metabólicos. Por su parte, en la UIM en Enfermedades Endocrinas se integró la Clínica Multidisciplinaria de Obesidad Mórbida, con más de 100 procedimientos bariátricos anualmente, $\mathrm{y}$ ha trabajado en el desarrollo de clínicas multidisciplinarias de enfermedades endocrinas complejas como cáncer de tiroides, acromegalia, Cushing, adenomas no funcionantes y diabetes tipo 1.

El Centro de Investigación Biomédica del Sur, ubicado en Xochitepec, Morelos, ha trabajado durante más de 30 años en la generación de fármacos a partir de plantas con propiedades medicinales. Partiendo de extractos de especies como Solanum chrysoticum y Ageratina pichinchensis, ha desarrollado antimicóticos. A partir de extractos de Hibiscus sabdariffa y Galphimia glauca desarrolló un antihipertensivo y un ansiolítico, respectivamente. Es importante destacar que en todos los casos se han realizado ensayos clínicos, con resultados prometedores. Otro desarroIlo biotecnológico importante es el logrado por la UIM en Inmunoquímica. Los investigadores de dicha unidad desarrollaron una vacuna experimental polivalente contra Salmonella, la cual fue probada en ensayos clínicos fase I, con resultados alentadores. Es importante destacar que este grupo de investigación se encuentra colaborando con otros grupos e instituciones, nacionales y extranjeras, en el desarroIlo de vacunas contra diversas enfermedades, entre ellas COVID-19.

En el Laboratorio de Oncoinmunología del CIBIOR se integró un programa de investigación enfocado a la incidencia en leucemia linfoblástica aguda infantil, que busca profundizar en la etiopatogenia de la enfermedad y contribuir con herramientas útiles para su diagnóstico, pronóstico y tratamiento. Para ello, se ha formado un grupo multidisciplinario y multiinstitucional de investigadores y médicos, encabezado por la doctora Rosana Pelayo, del CIBIOR, con el objetivo de contribuir a la disminución de la incidencia de esta enfermedad y a incrementar las tasas de supervivencia postratamiento.

El último ejemplo de trabajo de investigación que ha tenido impacto en la atención al derechohabiente corresponde al Banco de Células Hematopoyéticas de sangre de cordón umbilical, el cual fue creado a partir de la investigación que se realiza en la UIM en Enfermedades Oncológicas. Dicho banco se estableció en 2005 y, a la fecha, cuenta con aproximadamente 1500 unidades almacenadas, de las cuales más de 160 han sido utilizadas. Con dichas unidades se han llevado a cabo más de 120 trasplantes, principalmente en pacientes pediátricos, para el tratamiento de diversas enfermedades hematológicas, metabólicas y neoplásicas. ${ }^{65-67}$

\section{Retos y perspectivas futuras}

La investigación científica en el IMSS se ha enfocado en dos aspectos: generar conocimiento nuevo que ayude a entender el origen y la progresión de las enfermedades, y utilizar dicho conocimiento para mejorar el tratamiento y la atención integral al derechohabiente, así como apoyar a las autoridades en la toma de decisiones. El cambio constante en las tendencias epidemiológicas, el crecimiento de las poblaciones y los problemas de salud emergentes, aunados a la necesidad de trabajar con recursos financieros limitados, ha hecho necesario que las actividades de la investigación en salud en el IMSS se dirijan preferencialmente a la atención de los problemas prioritarios de salud; no obstante, no se debe perder el compromiso de seguir realizando investigación competitiva y seguir formando nuevas generaciones de investigadores que asuman el reto de producir conocimiento y de utilizarlo en beneficio de los derechohabientes.

La investigación en salud no es un lujo, sino una necesidad que todo país debe reconocer y asumir. Sin embargo, para realizar investigación de calidad, esta debe incorporar en su quehacer la preparación, aptitud y entusiasmo de los investigadores que la realicen, así como de las autoridades que la respalden. El compromiso de los primeros es reconocer las necesidades de investigación de manera crítica y realizarla de la mejor manera posible. El compromiso de los segundos es proporcionar las facilidades y los recursos necesarios para llevar a cabo las actividades de investigación.

La incorporación de la investigación en la atención a la salud en el IMSS ha sido un proceso gradual y continuo que, a la fecha, se traduce en una gran estructura científica, tanto humana como física, que se cuenta entre las más sólidas en México. Mantener, consolidar y fortalecer la estructura de investigación en el IMSS es, indudablemente, uno de los retos relevantes para conservar la calidad de la atención médica a los trabajadores de México.

\section{Reflexiones finales}

Desde los albores de la medicina, se concibió el ejercicio de la práctica médica como el resultado 
indisoluble del binomio médico-paciente. Hoy en día, las instituciones de salud más sólidas en el mundo han consolidado el trinomio médico-investigación-paciente. Su fundamento es que, como resultado de la investigación, el médico adquiera y aplique nuevos conocimientos, habilidades y tecnologías en beneficio de la salud de sus pacientes. En el ámbito de la medicina moderna, es fundamental entender la importancia del trinomio médico-investigación-paciente y, en consecuencia, se debe apoyar y fortalecer la estructura de la investigación en las instituciones de salud.

\section{Financiamiento}

La presente investigación no ha recibido ninguna beca específica de agencias de los sectores públicos, comercial o sin ánimo de lucro.

\section{Conflicto de intereses}

Los autores declaran no tener conflicto de intereses alguno.

\section{Responsabilidades éticas}

Protección de personas y animales. Los autores declaran que para esta investigación no se realizaron experimentos en seres humanos ni en animales.

Confidencialidad de los datos. Los autores declaran que en este artículo no aparecen datos de pacientes.

Derecho a la privacidad y consentimiento informado. Los autores declaran que en este artículo no aparecen datos de pacientes.

\section{Bibliografía}

1. Prentice T. Health, history and hard choices: Funding dilemmas in a fast-changing world. EE. UU.: World Health Organization/University of Indiana; 2006

2. Instituto Mexicano del Seguro Social. Contribuciones del IMSS a la medicina mundial. Pasado, presente y futuro. Instituto Mexicano del Seguro Social/Instituto Politécnico Nacional; 2018.

3. Armendares S, Salamanca F, Frenk S. Chromosome abnormalities in severe protein calorie malnutrition. Nature. 1971;232:271-273.

4. Armendares S, Buentello L, Salamanca F, Cantú-Garza JM. A dicentric $\mathrm{Y}$ chromosome without evidence of sex chromosomal mosaicism, 46,XYqdic, in a patient with features of Turner's syndrome. J Med Genet. 1972:9:96-100

5. Salamanca F, Buentello L, Sánchez J, Armendares S. A patient with 44 chromosomes. Ann Genet. 1985;28:130-132.

6. Cantú JM, García-Cruz D, Sánchez-Corona J, Hernández A, Nazara Z A distinct osteochondrodysplasia with hypertrichosis individualization of a probable autosomal recessive entity. Hum Genet. 1982;60:36-41.

7. Salcedo M, Piña-Sánchez P, Vallejo-Ruiz V, Monroy-García A, Aguilar-Lemarroy A, Cortés-Gutiérrez El, et al. Human papillomavirus genotypes among females in México: a study from the Mexican Institute for Social Security. Asian Pac J Cancer Prev. 2014;15:10061-1006.
8. Aguilar-Lemarroy A, Vallejo-Ruiz V, Cortés-Gutiérrez El, Salgado-Bernabé EM, Ramos-González NP, Ortega-Cervantes L, et al. Human papillomavirus infections in Mexican women with normal cytology, precancerous lesions, and cervical cancer: type-specific prevalence and HPV coinfections. J Med Virol. 2015:87:871-884.

9. Partida-Rodríguez O, Torres J, Flores-Luna L, Camorlinga M, Nieves-Ramírez M, Lazcano E, et al. Polymorphisms in TNF and HSP-70 show a significant association with gastric cancer and duodenal ulcer. Int $\mathrm{J}$ Cancer. 2010;126:1861-1868.

10. Pérez-Rodríguez M, Partida-Rodríguez $\mathrm{O}$, Camorlinga-Ponce M, Flores-Luna L, Lazcano E, Gómez A, et al. Polymorphisms in HLA-DQ genes, together with age, sex, and Helicobacter pylori infection, as potential biomarkers for the early diagnosis of gastric cancer. Helicobacter. 2017;22.

11. Chávez-González A, Ayala-Sánchez M, Sánchez-Valle $E$, Ruiz-Sánchez E, Arana-Trejo RM, Vela-Ojeda J, et al. Functional integrity in vitro of hematopoietic progenitor cells from patients with chronic myeloid leukemia that have achieved hematological remission after different therapeutic procedures. Leuk Res. 2006:30:286-295

12. Flores-Figueroa E, Arana-Trejo RM, Gutiérrez-Espíndola G, Pérez-Cabrera A, Mayani $\mathrm{H}$. Mesenchymal stem cells in myelodysplastic syndromes: phenotypic and cytogenetic characterization. Leuk Res. 2005;29:215-224.

13. Velázquez-Ávila $M$, Balandrán JC, Ramírez-Ramírez $D$, Velázquez-Avila M, Sandoval A, Felipe-López A, et al. High cortactin expression in B-cell acute lymphoblastic leukemia is associated with increased transendothelial migration and bone marrow relapse. Leukemia. 2019;33:1337-1348

14. Taniguchi-Ponciano K, Andonegui-Elguera S, Peña-Martínez E, Silva-Román G, Vela-Patiño S, Gómez-Apo E, et al. Transcriptome and methylome analysis reveals three cellular origins of pituitary tumors. Sci Rep. 2020:10:19373.

15. Muñoz-Ramírez Z, Pascoe B, Méndez-Tenorio A, Mourkas E, Sandoval-Motta S, Pérez-Pérez G, et al. A 500-year tale of co-evolution, adaptation, and virulence: Helicobacter pylori in the Americas. ISME J. 2020;15:78-92.

16. Muñoz-Ramírez ZY, Méndez-Tenorio A, Ikuko K, Bravo MM, Rizzato C, Thorell K, et al. Whole genome sequence and phylogenetic analysis show Helicobacter pylori strains from Latin America have followed a unique evolution pathway. Front Cell Infect Microbiol. 2017:7:50.

17. Torres-Juárez F, Cárdenas-Vargas A, Montoya-Rosales A, González-Curiel I, García-Hernández MH, Enciso-Moreno JA, et al. LL37 immunomodulatory activity during Mycobacterium tuberculosis infection in macrophages. Infect Immun. 2015;83:4495-4503.

18. Isibasi A, Ortiz V, Vargas M, Paniagua J, González C, Moreno J, et al. Protection against Salmonella typhi infection in mice after immunization with outer membrane proteins isolated from Salmonella typhi 9,12,d,Vi. Infect Immunol. 1988;56:2953-2959.

19. Blanco F, Isibasi A, González CR, Ortiz V, Paniagua J, Arreguín C, et al Human cell mediated immunity to porins from Salmonella typhi. Scand $J$ Infect Dis. 1993;25:73-80.

20. Chávez-Rueda K, Hernández J, Centeno E, Leano's-Miranda A, Legorreta-Haquet MA, Blanco-Favela F. Identification of prolactin as a novel immunomodulator on the expression of costimulatory molecules and cytokine secretions on $\mathrm{T}$ and $\mathrm{B}$ human lymphocytes. Clin Immunol. 2005:116:182-191.

21. López-Alarcon M, Villalpando S, Fajardo A. Breast-feeding lowers the frequency and duration of acute respiratory infection and diarrhea in infants under six months of age. J Nutra. 1997;127:436-443.

22. Villalpando S, López-Alarcón M. Growth faltering is prevented by breast-feeding in underprivileged infants from México City. J Nutra. 2000; $130: 546-552$

23. López Alarcón M. F-García M, del Valle O, González-Moreno G, Martínez-Basile A, Villegas R. Oral administration of docosahexaenoic acid attenuates interleukin- $1 \beta$ response and clinical course of septic neonates. Nutrición. 2012;28:384-390.

24. López-Alarcón M, Perichart-Perera O, Flores-Huerta S, Inda-Icaza P Rodríguez-Cruz M, Armenta-Álvarez, et al. Excessive refined carbohydrates and scarce micronutrients intakes increase inflammatory mediators and insulin resistance in prepubertal obese children independently of obesity. Mediators Inflame. 2014;2014:849031.

25. Barbosa-Cortes L, Villasís-Keever MA, del Prado-Manriquez $M$, López-Alarcón M. Adiposity and insulin resistance in children from a rural community in Mexico. Arch Med Res. 2015:46:214-220.

26. López-Alarcón M, Inda-Icaza P, Márquez-Maldonado MC, Armenta-Álvarez A, Barbosa-Cortés L, Maldonado-Hernández J, et al. A randomized control trial of the impact of LCPUFA- $\omega 3$ supplementation of body weight and insulin resistance in pubertal children with obesity. Pediatr Obes. 2019;14:e12499

27. López Alarcón MG Vital-Reyes VS, Hernández-Hernández FI, Maldonado-Hernández J. The role of LCPUFA $\omega 3$ on the obesity-associated hyperandrogenemia of pubertal girls: secondary analysis of a randomized clinical trial. J Pediatr Endocrino Meta. 2020;33:347-354. 
28. Cameron E, Martínez-Marina V, Chan A, Valladares A, Simmons L, Wacher N, et al. MGEA5-14 polymorphisms and type 2 diabetes in Mexico City. Am J Hum Biol. 2007;19:593-596.

29. Villarreal-Molina MT, Flores-Dorantes MT, Arellano-Campos O, Villalobos-Comparan M, Rodríguez-Cruz M, Miliar-García A, et al. Association of the ATP-binding cassette transporter A1 R230C variant with early-onset type 2 diabetes in a Mexican population. Diabetes. 2008;57:509-513.

30. Vázquez-Moreno M, Zeng H, Locia-Morales D, Peralta-Romero J, Asif H Maharaj A, et al. The melanocortin 4 receptor p.lle269Asn mutation is associated with childhood and adult obesity in Mexicans. J Clin Endocrinol Metab. 2020;105:dgz276.

31. Vázquez-Moreno M, Locia-Morales D, Valladares-Salgado A, Sharma T, Pérez-Herrera A, González-Dzib R, et al. The MC4R p.ILe269Asn mutation confers a high risk for type 2 diabetes in the Mexican population via obesity dependent and independent effects. Sci Rep. 2021:11:3097.

32. Cruz M, García-Macedo I, García-Valerio Y, Gutiérrez M, Medina-Navarro, R, Durán G, et al. Low adiponectin levels predict type 2 diabetes in Mexican children. Diabetes Care. 2004;27:1451-1453.

33. Ferreira-Hermosillo A, Molina-Ayala MA, Ramírez-Rentería C, Vargas G González B, Isibasi A. Inflammatory cytokine profile associated with metabolic syndrome in adult patients with type 1 diabetes. J Diabet Res. 2015;972073.

34. Villeda-González JD, Gómez-Olivares JC, Baiza-Gutman LA, Manuel-Apolinar L, Damasio-Santana L, Millán-Pacheco C, et al. Nicotinamide reduces inflammation and oxidative stress via the cholinergic system in fructose-induced metabolic syndrome in rats. Life Sci. 2020;250:117585

35. Zurita-Cruz J, Villasís-Keever M, Manuel-Apolinar L, Damasio-Santana L, Wakida-Kusunaki GH, Padilla-Rojas M, et al. Resistin/uric acid index as a prognostic factor in adolescents with obesity after lifestyle intervention. J Pediatr. 2020:219:38-42

36. Cadena-Obando D, Ramírez-Rentería C, Ferrera-Hermosillo A, Albarrán-Sanchez A, Sosa-Eroza E, Molina-Ayala M, et al. Are there really any predictive factors for a successful weight loss after bariatric surgery? BMC Endocr Disord. 2020;20:20.

37. Gómez-Zamudio JE, Mendoza-Zubieta V, Ferreira-Hermosillo A, Molina-Ayala M, Valladares-Salgado A, Suárez-Sánchez F, et al. High thyroid stimulating hormone levels increase proinflammatory and cardiovascular markers in patients with extreme obesity. Arch Med Res. 2016;47:476-482.

38. Espinosa E, Salame L, Marrero-Rodríguez D, Romero-Nieves AH, Cuenca D, Castelán-Martínez D, et al. Expression of the growth hormone receptor isoforms and its correlation with the metabolic profile in morbidly obese subjects. Endocrine. 2019;63:573-581.

39. Cervantes-Theruel J, Albarrán-Sánchez A, Rodríguez-Pérez V, Espinosa-Cárdenas E, Ramírez-Rentería C, Ferreira-Hermosillo A. Utility of the STOP-Bang and Epworth scales and the neck-to-height ratio to detect severe obstructive apnea-hypopnea syndrome in severe obesity. Surg Obes Rel Dis. 2021;17:257-262.

40. Guerrero-Romero F, Jaquez-Chairez FO, Rodríguez-Morán M. Magnesium in metabolic syndrome: A review based on randomized double blind clinical trials. Magnes Res. 2016;29:146-153.

41. Guerrero-Romero F, Flores-García A, Saldaña-Guerrero S, Simental-Mendía LE, Rodríguez-Morán M. Obesity and hypomagnesemia. Eur J Intern Med. 2016:34:29-33.

42. Simental-Mendía LE, Simental-Mendía M, Sahebkar A, Rodríguez-Morán M, Guerrero-Romero F. Effect of magnesium supplementation on lipid profile: a systematic review and meta-analysis of randomized controlled trials. Eur J Pharmacol. 2017;73:525-536.

43. Rodríguez-Morán M, Simental-Mendía LE, Gamboa-Gómez Cl, Guerrero-Romero F. Oral magnesium supplementation and metabolic syndrome: a randomized double-blind placebo-controlled clinical trial. Adv Chron Kidney Dis. 2018;25:291-296.

44. Guerrero-Romero F, Simental-Mendía LE, González-Ortiz M, Martínez-Abundis E, Ramos-Zavala, MG, Hernández-González SO, et al. The product of triglycerides and glucose, a simple measure of insulin sensitivity. Comparison with the euglycemic-hyperinsulinemic clamp. J Clin Endocrinol Metab. 2010;95:3347-3351.

45. Gómez-Diaz RA, Aguilar MV, Meguro EN, Márquez RH, Magaña EG, Martínez-García MC, et al. The role of natural killer T (NKT) cells in the pathogenesis of type 1 diabetes. Curr Diabetes Rev. 2011;7:278-283.

46. Ferreira-Hermosillo A, Ramírez-Rentería C, Molina-Ayala MA Mendoza-Zubieta V. Utility of the waist-to-height ratio, waist circumference and body mass index in the screening of the metabolic syndrome in adult patients with type 1 diabetes mellitus. Diabetol Metab Synd. 2014:6:32-42.

47. Paniagua R, Amato D, Vonesh E, Correa-Rotter R, Raos A, Morán J et al. Effects of increased peritoneal clearances on mortality rates in peritoneal dialysis: ADEMEX, a prospective, randomized, controlled trial. J Am Soc Nephrol. 2002;13:1307-1320.
48. Paniagua R, Amato D, Vonesh E, Mujais S, Horl WH, for the Mexican Nephrology Collaborative Study Group. Predictive value of natriuretic peptides in patients on peritoneal dialysis: results from the Ademex trial. Clin J Am Soc Nephrol. 2008;3:407-415.

49. Vicente-Martínez M, Martínez-Ramírez L, Muñoz R, Ávila M, Ventura MJ, Rodríguez $\mathrm{E}$, et al. Inflammation in patients on peritoneal dialysis is associated to increased extracellular fluid volume. Arch Med Res. 2004:35:220-224.

50. Ávila-Díaz M, Ventura MJ, Valle D, Vicente-Martínez M, García-González Z, Cisneros C, et al. Inflammation and extracellular volume expansion are related to sodium and water removal in patients on peritoneal dialysis. Perit Dial Int. 2006;26:574-580.

51. Prado-Uribe MC, Ventura MJ, Ávila-Díaz M, Mora CJ, Méndez-Durán A, Villanueva-Noches $\mathrm{D}$, et al. Low triiodothyronine is associated with $\mathrm{N}$-terminal pro-brain natriuretic peptide and mortality in dialysis patients. $\mathrm{Ne}$ frologia. 2017;37:598-607.

52. Sánchez V, Paniagua R, Prado MD, Mora C, Orihuela O, Martínez-Baca F, et al. High prevalence of diastolic dysfunction in incident patients on peritoneal dialysis: association with low thyroid hormones. Nephron. 2017;135:120-128.

53. Paniagua $R$, Ventura MJ, Avila-Diaz M, Hinojosa-Heredia H, Méndez-Durán $\mathrm{A}$, Cueto-Manzano $\mathrm{A}$, et al. NT-proBNP, fluid volume overload, and dialysis modality are independent predictors of mortality in ESRD patients. Nephrol Dial Transplant. 2010;25:551-557.

54. Jiménez-Rosales A, Amaya-Chávez A, Domínguez García MV, Camarillo-Romero E, Huitrón-Bravo GG, Majluf-Cruz A. Association of inflammatory and oxidative stress biomarkers in subjects with cardiovascular risk. Am J Ther. 2013:20:422-431.

55. Camarillo-Romero E, Domínguez-García MV, Amaya-Chávez A, Camarillo-Romero MS, Talavera-Piña J, Huitrón-Bravo G, et al. Effects of a physical activity program on markers of endothelial dysfunction, oxidative stress, and metabolic status in adolescents with metabolic syndrome. ISRN Endocrinol. 2012;2012:970629.

56. Rivera-García BE, Esparza-García JC, Aceves-Chimal JL, Leaños-Miranda A, Majluf-Cruz A, Isordia-Salas I. Platelet glycoprotein IIla PIA1/ A2 polymorphism in young patients with ST elevation myocardial infarction and idiopathic ischemic stroke. Mol Cell Biochem. 2013;384:163-171.

57. Isordia-Salas I, Santiago-Germán D, Cerda-Mancillas MC, Hernández-Juárez J, Bernabé-García M, Leaños-Miranda A, et al. Gene polymorphisms of angiotensin-converting enzyme and angiotensinogen and risk of idiopathic ischemic stroke. Gene. 2018;688:163-170

58. Camacho-Mejorado R, Gómez R, Torres-Sánchez LE, Hernández-Tobías EA, Noris G, Santana C, et al. AOX5, LPA, MMP9 and TPO gene polymorphisms increase atherothrombosis susceptibility in middle-aged Mexicans. R Soc Open Sci. 2020:6:190775.

59. Proaño JV, Madrazo I, Avelar F, López-Félix B, Díaz G, Grijalva I. Medical treatment for neurocysticercosis characterized by giant subarachnoid cysts. N Engl J Med. 2001:345:879-885.

60. Madrazo I, Drucker-Colín R, Díaz V, Martínez-Mata J, Torres C, Becerril JJ. Open microsurgical autograft of adrenal medulla to the right caudate nucleus in two patients with intractable Parkinson's disease. N Engl J Med. 1987;316:831-834.

61. Madrazo I, León V, Torres C, Aguilera MC, Varela G, Álvarez F, et al. Transplantation of fetal substantia nigra and adrenal medulla to the caudate nucleus in two patients with Parkinson's disease. N Engl J Med. 1988;318:51.

62. Grijalva I, Guízar-Sahagún G, Castañeda-Hernández G, Mino D, Maldonado-Julián $\mathrm{H}$, Vidal-Cantú $\mathrm{G}$, et al. Efficacy and safety of 4-aminopyridine in patients with long-term spinal cord injury: a randomized, double-blind, placebo-controlled trial. Pharmacotherapy. 2003;23:823-834.

63. Grijalval, García-Pérez A Díaz J, Aguilar S, Mino D, Santiago-Rodríguez E, et al. High doses of 4-aminopyridine improve functionality in chronic complete spinal cord injury patients with MRI evidence of cord continuity. Arch Med Res. 2010;41:567-575.

64. Buzoianu-Anguiano V, Rivero-Osorio J, Orozco-Suárez S, Vega-García A García-Vences E, Sánchez-Torres S, et al. Single versus combined therapeutic approaches in rats with complete chronic spinal cord injury. Front Neurol. 2020:11:136-140.

65. Novelo-Garza B, Limón-Flores A, Guerra-Márquez A, Luna-Bautista F, Juan-Shum L, Montero I, et al. Establishing a cord blood banking and transplantation program in Mexico: a single institution experience. Transfusion. 2008:48:228-236.

66. Guerra Márquez A, Novelo-Garza B, Malagón-Martínez A, Limón-Flores A, Luna-Bautista F, Juan-Shum L, et al. Cord blood banking and transplanation at the Mexican Institute of Social Security: the first five years. Transfusion. 2011;51:328-332.

67. Guerra-Márquez A, Peñaflor K, Mayani H. Cord blood banking and transplantation at the Mexican Institute of Social Security: thirteen years of experience. Arch Med Res. 2020;51:54-62. 